\title{
Low Losses Printed Distribution Network Technologies for Planar Antennas in Ka Band
}

\author{
Adrián Tamayo-Domínguez , José-Manuel Fernández-González , Manuel Sierra-Pérez
}

\begin{abstract}
Study of Substrate Integrated Waveguide and Gap Waveguide technologies used to manufacture some demonstration prototypes at $\mathrm{Ka}$ band for a low losses distribution network in a low profile antenna. A transmission losses comparison is presented using lossy and lossless dielectric substrates in the simulations for the different technologies at $\mathbf{3 0}$ GHz. Various structures such as transitions from WR-28, bends, and power dividers have been designed and simulated. The simulations of different models are compared with the manufactured prototypes in metallized 3D-printed plastic technology and in RO4350B and FR-4 substrates for a band from 28 to $30 \mathrm{GHz}$.
\end{abstract}

Index Terms-low profile antenna, Ka band, $30 \mathrm{GHz}$, printed technologies, transmission losses, substrate integrated waveguide, ridge gap waveguide, groove gap waveguide, distribution network, RO4350B, FR-4, metallized 3D-printed plastic.

\section{INTRODUCTION}

One of the most critical aspects in planar antennas at frequencies from $\mathrm{Ka}$ band and above is the losses in the distribution network. This is because the greater the losses the bigger the number of radiant elements needed and then, the size and cost of the antenna. In order to achieve very low losses while keeping a good manufacturing performance in cost, weight and size, Substrate Integrated Waveguide (SIW) and Gap Waveguide (GW) technologies have been considered [1], [2]. The SIW technology involves using a dielectric substrate where top and bottom plates are connected through two periodic rows of metallized via holes that behave like the walls of the guide, so that a path in which the wave propagates is obtained [1], [3]. As for the GW technology, there are two types of possible structures: with ridge (RGW) and with groove (GGW). RGW uses a waveguide with a central ridge on which the lateral walls are replaced by a metamaterial surface. This surface prevents the transmission of power in the transverse direction and confines the wave above the central ridge [2], [4]. In GGW the central ridge is deleted, having an emulated rectangular waveguide filled with air and with lateral metamaterial walls [2]. Using these types of new technologies, distribution networks with very low losses at high frequencies are expected to obtain.

\section{SIW AND GW TRANSMISSION LOSSES}

First, a study has been performed considering transmission losses for SIW and GW technologies designed for working in $30 \mathrm{GHz}$ using different dielectric constants and lossy or lossless substrates. In addition, the same study has been conducted for dielectric-filled rectangular waveguide and microstrip in order to compare the results obtained. Design expressions for SIW can be found at [1], and a procedure for RGW design is explained in [5]. For GGW, the design of the metamaterial walls is similar to the one used in RGW and the width is adjusted for working at the desired band.

The models that have been simulated present the structures shown in Fig. 1, where the air gap in GGW and $\mathrm{RGW}$ is between upper metallic plate and the dielectric surface in blue. GGW also presents air within the groove.

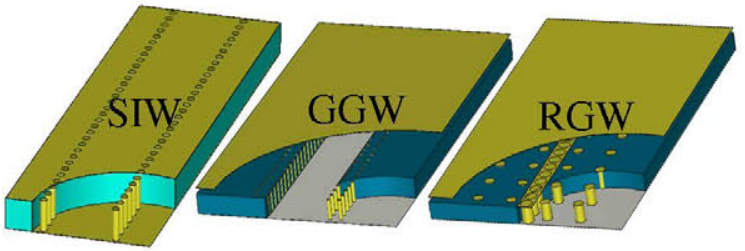

Fig. 1: Structures for SIW, GGW and RGW.

Transmission losses using substrates with dielectric constant $\varepsilon_{\mathrm{r}}$ from 1 to 8.5 and loss tangent of 0 and 0.002 are shown in Fig. 2 and Fig. 3. Conductor losses in these technologies depend on the height of the substrate due to conduction effect on the metal, which is copper with a conductivity $\sigma=5.8 \cdot 10^{7} \mathrm{~S} / \mathrm{m}$, so that a constant relation has been used in the simulations. In SIW and rectangular waveguide (WG), height is half of the width of the guide. For RGW and GGW, the height $d$ of the pins in the metamaterial and the thickness of the air gap $h$ are related by the constant expression $h /(h+d)$ equals to 0.22 . For microstrip (MS), 50 ohm structures have been simulated with the same dielectric thicknesses used in SIW.

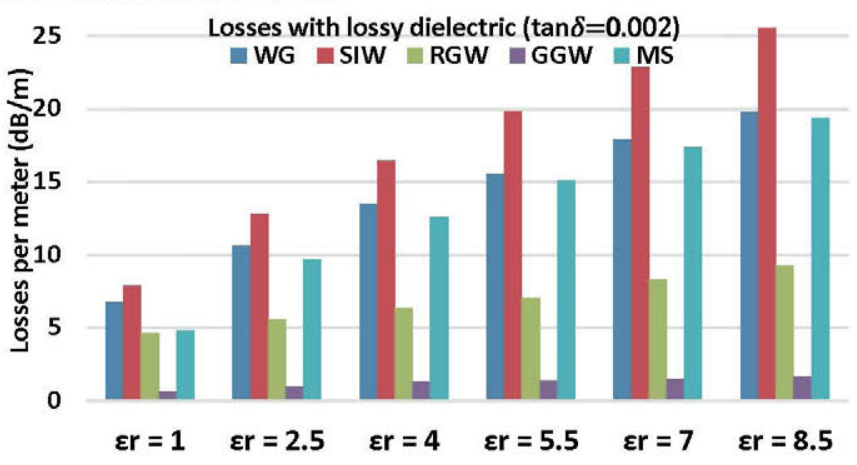

Fig. 2: Dielectric losses comparison $(\tan \delta=0.002)$.

With respect to the losses due to dielectric in Fig. 2, the expected results are obtained. SIW presents the highest dielectric losses due to the amount of dielectric inside the structure, as well as the rectangular waveguide. Rectangular waveguide has lower loses in the representation because the 
simulation includes the losses due to conductors, which are higher in SIW as can be seen in Fig. 3. Gap Waveguide technology shows its great performance in terms of losses when a lossy substrate is employed, especially Groove Gap Waveguide technology. In GGW, the impact of the dielectric losses is minimum due to the propagation occurs inside the air groove in the substrate. MS presents lower losses than SIW with the same substrate. However, the problem in microstrip above $\mathrm{X}$ band is the propagation of several surface modes that degrade the performance of the microstrip. Those surface modes can affect the behaviour of other components in the circuit, as well as an important coupling between radiating elements in a microstrip patch antenna or radiation losses. CPW is commonly used to avoid the problems of microstrip at high frequencies, but the attenuation is much higher than in microstrip. Moreover, the mode of propagation in CPW can degenerate into an asymmetric coupled slot-line mode or into parallel plate modes if conductor-backed CPW is used [6].

The same structures have been simulated considering a dielectric substrate without losses $(\tan \delta=0)$, which means that the losses obtained correspond only to conduction effects in the copper surfaces.

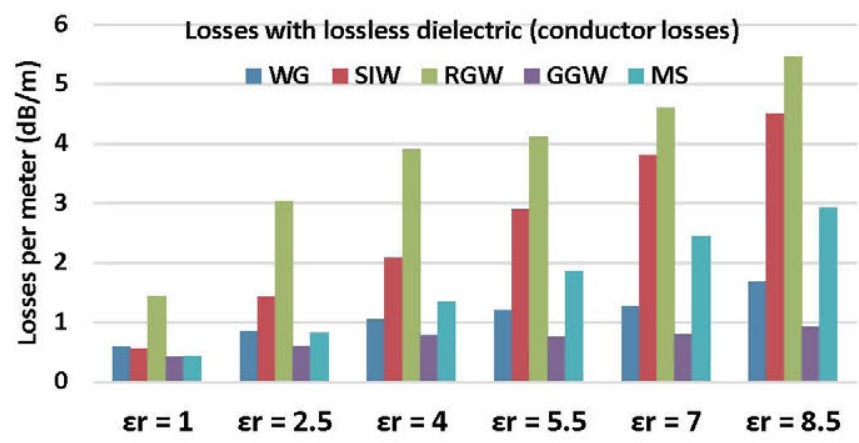

Fig. 3: Conductor losses comparison $(\tan \delta=0)$.

In Fig. 3 the losses in the RGW structure can be appreciated, followed by losses in SIW. Both structures present high conductor losses, the first one due to the ridge and the second one due to the via holes. Those losses increase when relative dielectric constant increases because the thickness of the substrate in SIW and the air gap in RGW are related to the width of SIW and dielectric thickness in RGW respectively. Therefore, as dielectric constant increases, both parameters decrease, so the current density is higher and this supposes the increment of ohmic losses. Rectangular waveguide presents lower conductor losses than SIW. The main part of the current intensity in a rectangular waveguide is found in the lateral walls. When these walls are substituted by via holes in SIW, the currents generated in the vias are more intense and it supposes more losses because those vias are very narrow. In the case of gap technology, it deletes the electric contact between upper and lower metal plates, so currents in the lateral pins are low. This reduces the conductor losses considerably but, in the case of RGW, the current density in the central ridge is very high and this increases notably the conductor losses. GGW is the technology with lowest ohmic losses because the ridge is deleted and top and bottom metal plates are not in contact, so intensity of currents outside the metal plates at the top and bottom of the groove area is low. With respect to MS, it present high conductor losses, but they are lower than for SIW and RGW due to the absence of via holes or a metal ridge between pins.

\section{MANUFACTURING TECHNOLOGIES.}

With all the considerations about transmission losses in each technology in Section II, the manufacturing processes and materials used for the design of prototypes with the lowest possible losses are selected. The final structures are oriented to a design with most of the energy propagating through air, which provides the lowest losses possible.

\section{A. Printed circuits with PTFE and FR-4}

The multilayer possibility in printed circuits allows designing hollow structures under certain conditions. This is the base consideration for the modelled and simulated SIW prototypes, because dielectric losses are still large although loss tangent is around 0.002. The idea consists on manufacturing SIW prototypes stacking three layers of materials: two thin substrate layers in the upper and lower faces of the SIW, which support the top and bottom copper layers, and a central layer that is cut to generate a hollow structure within most of the energy propagates.

That structure presents much lower losses due to propagation in air and means that material with very good properties are not extremely necessary. As the surrounding substrate is used like a support of the central hollow SIW, this offers the possibility of using materials with worse properties, which are more economical. For the first prototype, RO4350B and FR-4 substrates have been chosen. RO4350B is a relatively economical substrate used typically for lower frequency ranges or not critical losses applications. FR-4 substrate is commonly used for PCB and low frequency applications. It presents high losses and its properties strongly change between manufacturers and series, but it is the most economic option. Because of that, it has been taken into account for the design process as support material.

\section{B. Metallized 3D-printed plastic structures}

The rise of 3D printing and continuous development of the necessary technology has boosted the manufacture of various professional designs for both prototyping and mass production. Stimulation of this technology has had a major impact on much sectors of industry, including the field of radiocommunications and antenna design. Accompanying 3D printing, the necessary technology has been developed for metallizing models printed in various plastic materials. This provides great advantages over existing metal machining technology, which are: ease of manufacture, reduced costs, reduced lead times, reduced weight, increase in RF design flexibility and plastic provides better thermal insulation than metal.

For the interests of this work, metallized 3D-printing is oriented to the design of structures using air as substrate material. Gap Waveguide is the suitable technology for manufacturing in metallized 3D, because it is not needed an electrical connection between upper and lower metallic plates and the manufacture of the two parts can be made separately. The critical piece is the one that includes the periodic lateral pins, which presents a complicated structure that can be printed and metallized very easily.

\section{SIW DESIGN IN RO4350B AND FR-4}

A trade-off solution in printed circuit technology that combines low losses and reduction of cost is the combination of Rogers and FR-4 materials. Rogers RO4350B substrate is 
used as central layer because its losses are much lower than in FR-4 and the effect of the additional material in the margins of lateral via holes will be lower. FR-4 is used as support material for upper and lower copper plates, that are joint with the central RO4350B using prepreg for FR-4, which is economic and compatible between FR-4 and RO4350B substrates. This structure is shown in Fig. 4 and the main properties and parameters of this base structure are in Tables I and II.

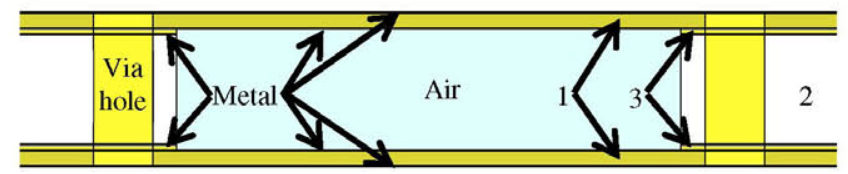

Fig. 4: SIW base structure.

TABLE I. MATERIALS OF SIW BASE STRUCTURE

\begin{tabular}{|c|c|c|c|}
\hline ID & Material & $\varepsilon_{\mathrm{r}}$ & $\tan \delta$ \\
\hline 1 & FR-4 0.2 mm & $4.8(1 \mathrm{MHz})$ & $0.014(1 \mathrm{MHz})$ \\
\hline 2 & RO4350B $1.524 \mathrm{~mm}$ & $3.66(10 \mathrm{GHz})$ & $0.0037(10 \mathrm{GHz})$ \\
\hline 3 & Prepreg FR-4 $0.065 \mathrm{~mm}$ & $4.8(1 \mathrm{MHz})$ & $0.014(1 \mathrm{MHz})$ \\
\hline
\end{tabular}

TABLE II. PARAMETERS OF SIW BASE STRUCTURE

\begin{tabular}{|c|c|}
\hline Parameter & Value \\
\hline Diameter of the vias & $0.8 \mathrm{~mm}$ \\
\hline Margin between via and border of material & $0.3 \mathrm{~mm}$ \\
\hline Total thickness (+metal) & $2.156 \mathrm{~mm}$ \\
\hline
\end{tabular}

In the structure of Fig. 4, all the copper plates are present in both faces in each substrate layer because it eases the manufacturing process due to the better adherence between copper surfaces than between substrate surfaces. The total thickness of the prototype will be $2.156 \mathrm{~mm}$, but the real height of the effective SIW model will be $1.688 \mathrm{~mm}$ due to the upper and lower layers of FR-4 are only support material and do not affect the behaviour of the guide. The structure and separation of via holes is shown in Fig. 5 and the parameters used for the desired frequency band from 28 to 30 $\mathrm{GHz}$ are presented in Table III.

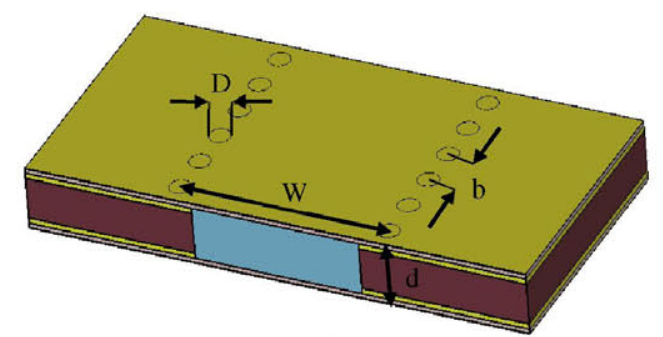

Fig. 5: Straight SIW section.

TABLE III. DESIGN PARAMETERS FOR SIW MODEL

\begin{tabular}{|c|c|c|c|}
\hline Parameter & Value & Parameter & Value \\
\hline $\mathrm{D}$ & $0.8 \mathrm{~mm}$ & $\mathrm{~b}$ & $1.6 \mathrm{~mm}$ \\
\hline $\mathrm{W}$ & $8 \mathrm{~mm}$ & $\mathrm{~d}$ & $2.156 \mathrm{~mm}$ \\
\hline
\end{tabular}

The cut-off frequencies for the first and second modes in the SIW model can be found in Table IV. The resultant bandwidth corresponds to the single mode frequencies. The desired band from 28 to $30 \mathrm{GHz}$ is included in the resultant bandwidth.

TABLE IV. CUT-OFF FREQUENCIES FOR SIW MODEL

\begin{tabular}{|c|c|c|}
\hline & Mode 1 & Mode 2 \\
\hline Cut-off frequency & $19.94 \mathrm{GHz}$ & $39.24 \mathrm{GHz}$ \\
\hline
\end{tabular}

Simulated and measured results are presented in Section VI together with GGW model shown in Section V.

\section{GGW DESIGN IN METALLIZED 3D-PRINTED PLASTIC}

The design of Groove Gap Waveguide models has been implemented in 3D-printed technology plated with a $10 \mu \mathrm{m}$ copper layer. The printing technology used is called stereolithography (SLA) and the accuracy of the final printed piece is specified as $30 \mu \mathrm{m}$ in $\mathrm{X}-\mathrm{Y}$ plane and $100 \mu \mathrm{m}$ in $\mathrm{Z}$ direction due to the stacking of plastic layers. The objective is to obtain the equivalent designs of the SIW models and compare the results. The base structure includes a bed of nails made of three rows of pins for the lateral metamaterial. This structure is shown in Fig. 6 and 7 and the design parameters are in Table V.

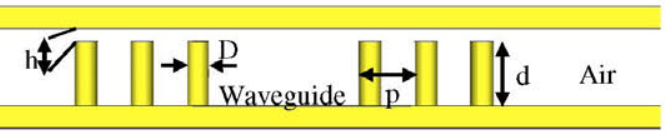

Fig. 6: GGW transversal structure for metallized 3D-printed plastic.

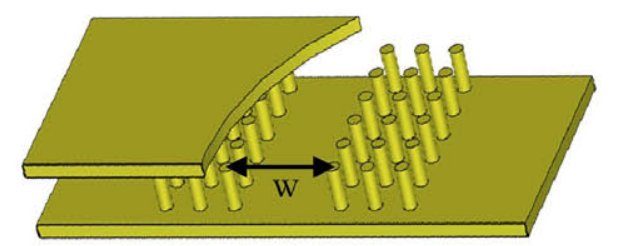

Fig. 7: GGW structure for metallized 3D-printed plastic.

TABLE V. PARAMETERS OF GGW BASE STRUCTURE

\begin{tabular}{|c|c|c|c|}
\hline Parameter & Value & Parameter & Value \\
\hline $\mathrm{h}$ & $0.6 \mathrm{~mm}$ & $\mathrm{p}$ & $2.6 \mathrm{~mm}$ \\
\hline $\mathrm{D}$ & $1 \mathrm{~mm}$ & $\mathrm{~d}$ & $3 \mathrm{~mm}$ \\
\hline $\mathrm{W}$ & $8 \mathrm{~mm}$ & Materials & Copper/Air \\
\hline
\end{tabular}

The start and end frequencies of the single mode band are presented in Table VI. As can be seen, this single mode band includes the desired frequency band.

TABLE VI. CUT-OFF FREQUENCIES FOR GGW MODEL

\begin{tabular}{|c|c|c|}
\hline Single mode band & Start & End \\
\hline Frequency & $23.02 \mathrm{GHz}$ & $38.10 \mathrm{GHz}$ \\
\hline
\end{tabular}

The losses per unit length have been calculated for SIW and GGW structures. In GGW, all the contribution comes from the conductor losses in the structure because the surrounding dielectric is lossless air. In Fig. 8, a comparison between losses in SIW and in GGW has been conducted. Losses in GGW, which are approximately $0.92 \mathrm{~dB} / \mathrm{m}$, are much lower than in SIW. There is a difference of $1 \mathrm{~dB} / \mathrm{m}$ between technologies, being the losses in GGW less than a half of the losses in SIW for the structures implemented.

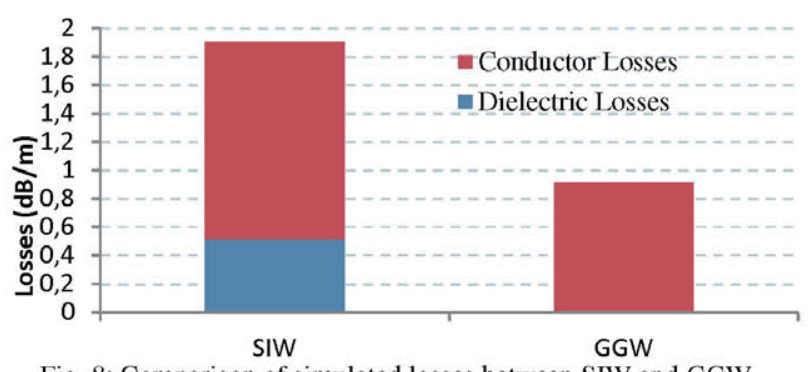

Fig. 8: Comparison of simulated losses between SIW and GGW. 


\section{FINAL DESIGNS AND RESULTS}

The final models that have been manufactured are presented in this section including the simulated results. The total length from inputs to outputs is $90 \mathrm{~mm}$. Two photos of the prototypes in SIW and in GGW are included in Fig. 9 and Fig. 10 respectively.

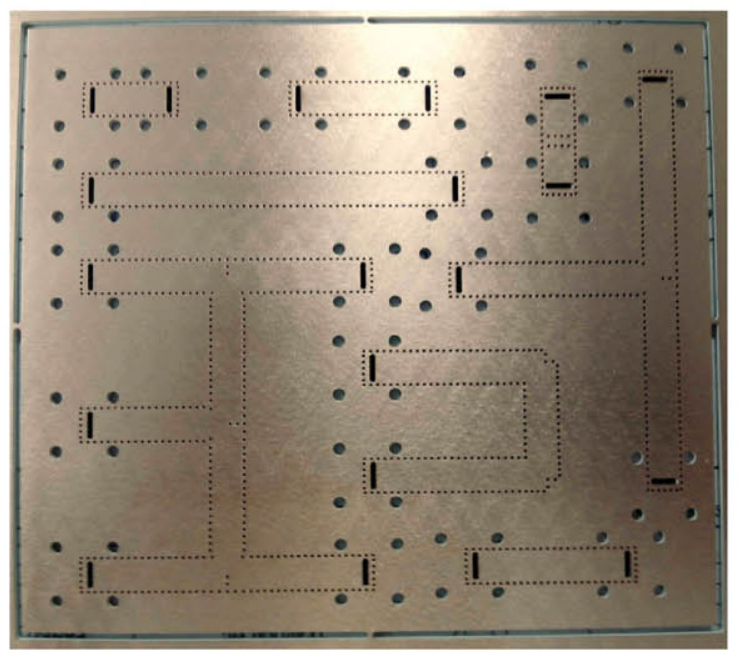

Fig. 9: Photo of the manufactured SIW prototype.

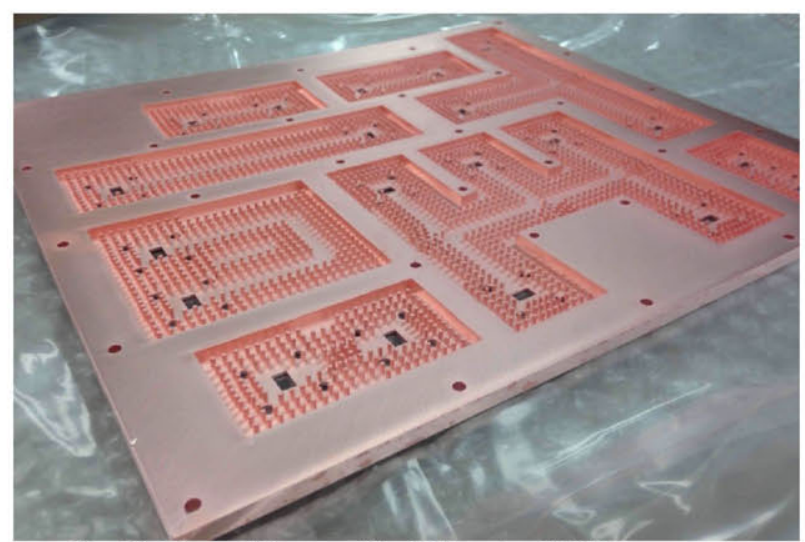

Fig. 10: Photo of the metallized 3D-printed GGW prototype.

In this paper, only simulations and measurements for the straight direct prototypes are presented in each technology.

\section{A. Direct SIW}

The results obtained in the simulation for the model in Fig. 11 are shown in Fig. 12, which are compared with the measurement of the real prototype. It can be observed that there is an important deviation between simulation and measurement in the form of the $\mathrm{S}$ parameters.

It has been found that the origin of that deviation is the shape of the manufactured transition. The simulated WR-28 to SIW transition presents a rectangular form, but the ends of the transition of the real prototype are rounded. Both structures for the transition are represented in Fig. 13.

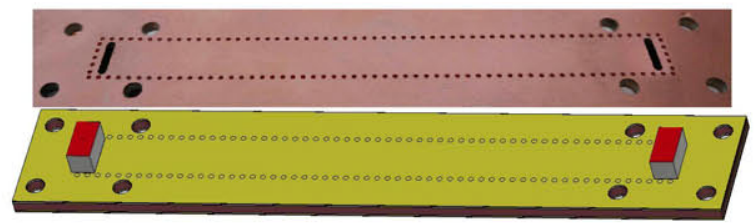

Fig. 11: Direct SIW prototype.

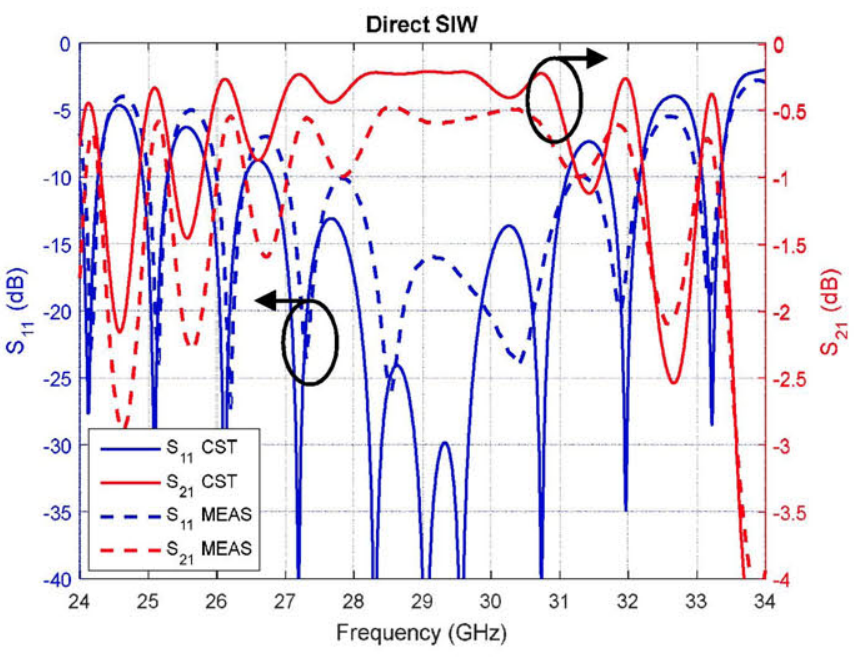

Fig. 12: Measure and simulation of the direct SIW prototype. Desired transition.

A new simulation has been performed using the manufactured structure of the transition in Fig. 13 and the final comparison between simulation and measurement is shown in Fig. 14.

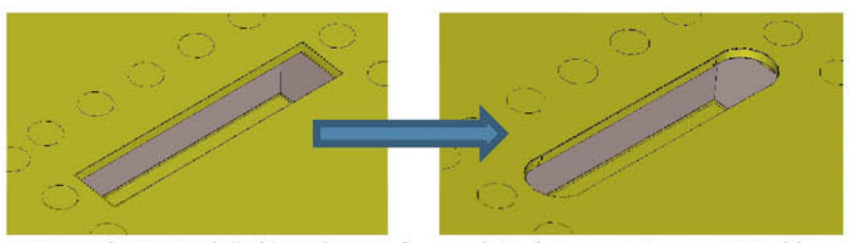

Fig. 13: Desired (left) and manufactured (right) transitions to WR-28.

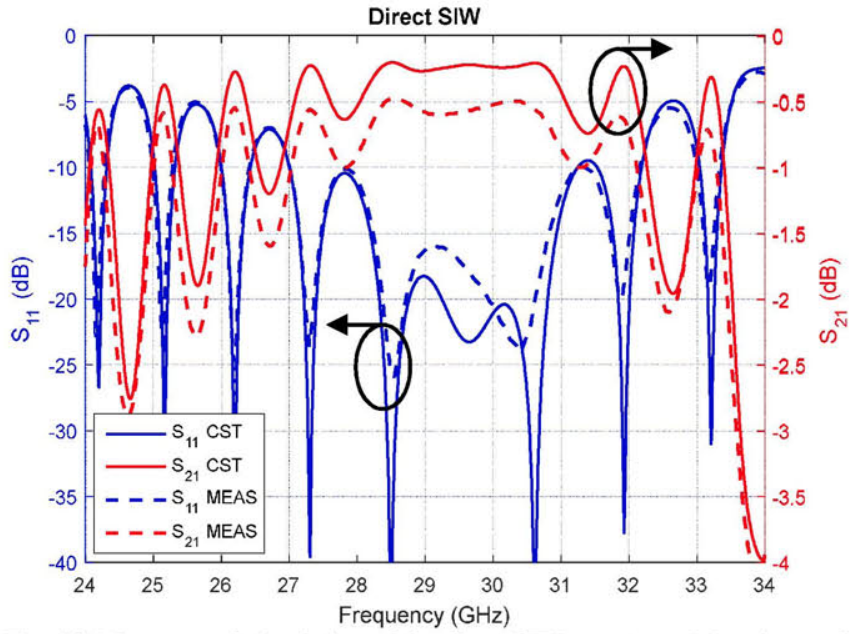

Fig. 14: Measure and simulation of the direct SIW prototype. Manufactured transition.

The similitude between simulation and measurement with the correction in the shape of the transition is very good, but it can be observed that the final losses are significantly higher than the simulated ones. The simulated losses are around 0.25 $\mathrm{dB}$ while the results after measurement show an average loss of $0.55 \mathrm{~dB}$. This difference is mainly caused by the roughness of the copper surfaces inside the structure. Higher losses in the central dielectric substrate may affect, but considering the simulated relation between conductor and dielectric losses in Fig. 8, its influence will be small. The deviation between desired and obtained results can be corrected with a reoptimization of the transition considering the rounded laterals in order to achieve a better matching in the desired frequency band. 


\section{B. Direct $G G W$}

The results obtained in the simulations and measurements for the GGW model in Fig. 15 are shown in Fig. 16. In this case, the similitude between both results is much better than in SIW because the edges in the transitions of the manufactured model present a great level of accuracy.

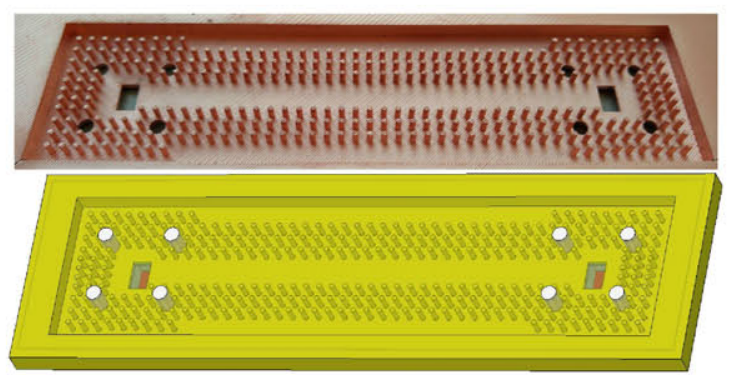

Fig. 15: Direct GGW prototype.

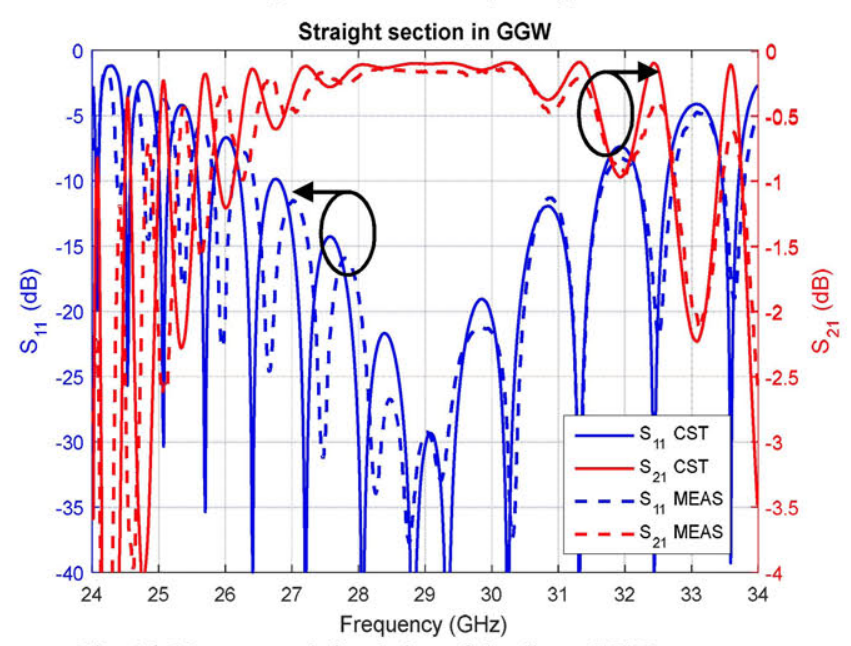

Fig. 16: Measure and simulation of the direct GGW prototype.

The measured losses in this prototype are approximately $0.16 \mathrm{~dB}$ in the desired band, which are very close to the simulated results of $0.12 \mathrm{~dB}$. Return losses are better than 15 $\mathrm{dB}$ between 28 and $30 \mathrm{GHz}$ and above $20 \mathrm{~dB}$ in most of the band. There is a slightly displacement in frequency that can be produced by manufacturing imperfections. In particular, the flat and wide structure of the plastic piece has forced to build the part at angle in order to avoid curved surfaces due to material shrink. This transfers the accuracy in $\mathrm{Z}$ axis (100 $\mu \mathrm{m})$ of the stacked layers along all surfaces and print lines become visible. This can be observed in the image in Fig. 17.

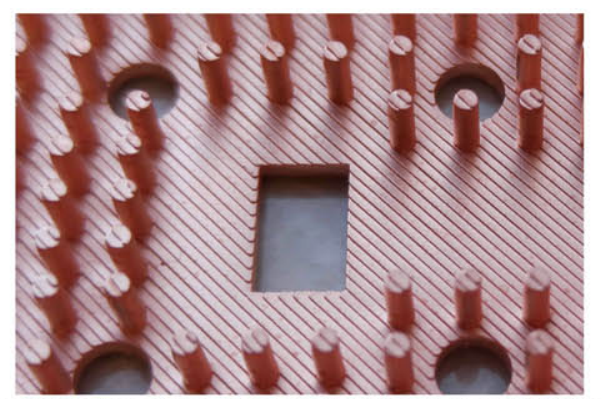

Fig. 17: Detail of the printed layers in the manufactured GGW prototype.

\section{Comparison of losses between SIW and GGW prototypes}

The average losses in the measurements for the prototypes in SIW and GGW are compared in Fig. 18. The relation of losses between SIW and GGW is similar to the simulations in
Fig. 8 with a visible increment of losses in SIW due to the effect of the dielectric substrate. Both values include the guide with a length of $90 \mathrm{~mm}$ and two transitions to WR-28.

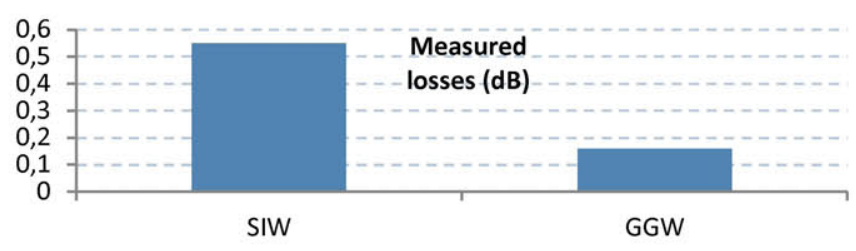

Fig. 18: Comparison of measured losses between SIW and GGW.

\section{CONCLUSIONS}

A losses comparison has been performed between SIW, RGW and GGW considering a substrate with dielectric constant and loss tangent as parameters. The best results have been achieved by SIW and GGW with an air-like substrate as expected. Prototypes in SIW using Rogers and FR-4 substrates with an air gap inside the structure have been simulated and measured, as well as GGW prototypes in metallized 3D-printed plastic. Final results provide a good reflection level in the desired band in both cases, although there is a mayor deviation in SIW due to transitions. Final simulations and measurements fit well, with losses of around $0.55 \mathrm{~dB}$ in SIW and $0.16 \mathrm{~dB}$ in GGW.

\section{ACKNOWLEDGMENT}

Simulations done in this work have been performed using CST Microwave Studio Suite 2016 under a cooperation agreement between Computer Simulation Technology (CST) and Technical University of Madrid. The authors want to acknowledge the Spanish Government, Ministry of Economy, National Program of Research, Development and Innovation for the support of the project ENABLING5G "Enabling Innovative Radio Technologies for 5G networks" (Project number TEC2014-55735-C3-1-R), associated to the FPI grant with reference BES-2015-075230. This study has been made under the Jetstream project, supported by the call "Retos Colaboración" from the Spanish "Ministerio de Economía" (RTC-2015- 3495-7) and with the collaboration of the company Telnet.

\section{REFERENCES}

[1] M. Bozzi, L. Perregrini, K. Wu and P. Arcioni, "Current and future research trends in substrate integrated waveguide technology," Radioengineering, 2009, vol. 18, no 2, p. 201-209.

[2] P.-S. Kildal, "Three metamaterial-based gap waveguides between parallel metal plates for $\mathrm{mm} / \mathrm{submm}$ waves," in Antennas and Propagation, 2009. EuCAP 2009. 3rd European Conference on, Mar. 2009, pp. 28-32.

[3] T. Djerafi and K. Wu, "Substrate integrated waveguide (SIW) techniques: The state-of-the-art developments and future trends," Journal of University of Electronic Science and Technology of China, 2013, vol. 42, no 2, p. 171-192.

[4] E. Alfonso, M. Baquero, P.-S. Kildal, A. Valero-Nogueira, E. RajoIglesias, and J. I. Herranz, "Design of microwave circuits in ridgegap waveguide technology," in Proc. IEEE MTT-S Microw. Symp. Dig., May 2010, p. 1544-1547.

[5] A. Tamayo, J.M. Fernández, J.M. Inclán, M. Sierra. "Design proposal for Ridge Gap Waveguide and comparison with other technologies in $\mathrm{Ka}$ to W bands", Proc. 10th EuCAP, DOI: 10.1109/EuCAP.2016.7481554.

[6] G. Poesen, "Towards an Adaptable Millimeter Wave Reflector: Development of an Antenna Coupled Opto-electronic Modulator Array", ASP/VUBPRESS/UPA, 2009. 